\title{
Tweezergate: A Cautionary Tale about Sample Preparation
}

\author{
Abigail P. Lindstrom, Nicholas W. M. Ritchie and Dale E. Newbury \\ National Institute of Standards and Technology, Gaithersburg, MD 20899-8371
}

It is common at meetings and in journal articles to discuss successes and rare to admit to mistakes or failures. In contrast, the authors wish to present a cautionary tale about what can go wrong, and to present just how important careful sample preparation, knowing your tools and the diligent use of control samples can be. A routine analysis of environmental particles using automated scanning electron microscope based energy dispersive X-ray spectrometry (SEMEDS) produced some atypical particle compositions. Normally, unusual particles are of interest in such samples. Unfortunately, our initial excitement cooled when the analysis of the control sample showed similar particles. Both the sample and the control had been prepared by dabbing a carbon sticky tab mounted on an aluminum pin mount on the surface of a cotton wipe. The pin mount was analyzed using SEM-EDX automated particle analysis using a 3-second x-ray acquisition live-time (see Fig 1.) The particles of interest were unusual due to high Co content, which is typically not found in ordinary dust and soil samples. Based on the average composition results from the automated particle analysis, an excellent match for the alloy was found using a Google search. The particles were likely Elgiloy [1], a non-magnetic and corrosion resistant industrial alloy. Based on the analysis of the control, it was assumed that the particles were introduced somewhere in the sample preparation process. Further investigation showed that they were an excellent match for the tweezers that were used to manipulate both the sample and the control wipe during preparation (see Fig 2.) When investigated, it was discovered that the alloy used to manufacture the tweezers had changed between batches. A slightly different model with the same geometry, but of a different alloy, had been ordered. Nevertheless, the tweezers had been cleaned following our routine protocol before use by sonication in ethanol and it was assumed that they were clean. Further investigations revealed that sonication was not particularly effective in removing spalling particles from the surface of the tweezers. A variety of solvents and different cleaning procedures were tried, but none was able to fully eliminate the contamination.

The sample preparation process requires the use of tweezers but these tweezers were a particularly poor choice because the tweezer's alloy was an alloy that would have been of interest in the analysis. If it is not possible to totally eliminate contamination from tools used in the sample preparation process, an alternative approach is to select tools such that the particles they generate will not be mistaken for particles of interest and to know the materials of the tools so they can be eliminated from the data set. The importance of control samples and careful analysis of specimen blanks cannot be understated, as they were paramount to identifying this source of measurement error.

\section{References}

[1] J. P. Frick, Ed, Woldman's Engineering Alloys, $7^{\text {th }}$ ed. (ASM International, Materials Park, $\mathrm{OH}, 1990) 425$. 


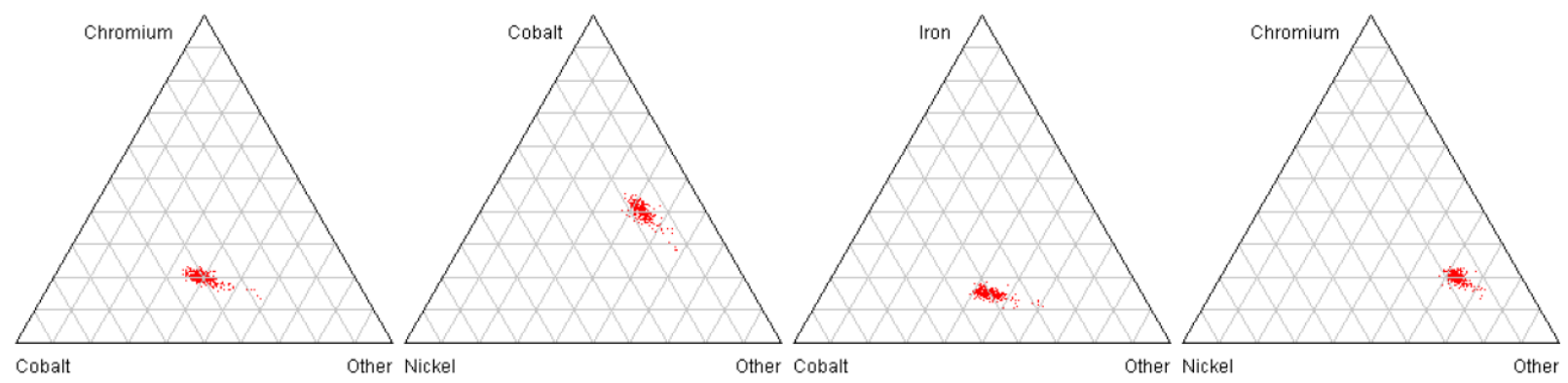

Fig 1: Ternary Diagrams showing the measured composition of the Elgiloy Particles



Fig 2: Comparing a spectrum collected from a contamination particle with a spectrum collected from the tweezers 